\title{
Inverse Agreement Algorithms with Application to Swarm Dispersion for Multiple Nonholonomic Agents
}

\author{
Dimos V. Dimarogonas and Kostas J. Kyriakopoulos
}

\begin{abstract}
We propose an inverse agreement control strategy for multiple nonholonomic agents that forces the team members to disperse in the workspace in a distributed manner. Both the cases of an unbounded and a circular bounded workspace are considered. In the first case, we show that the closed loop system reaches a configuration in which the minimum distance between any pair of agents is larger than a specific lower bound. It is proved that this lower bound coincides with the agents' sensing radius. In the case of a bounded workspace, the control law is modified to force the agents to remain within the workspace boundary throughout the closed loop system evolution. Moreover the proposed control guarantees collision avoidance between the team members. The results are supported through relevant computer simulations.
\end{abstract}

\section{INTRODUCTION}

The emerging use of multi-robot and multi-vehicle systems in various applications has recently raised the need for control designs that force a team of multiple vehicles/robots (from now on called "agents") to achieve various goals. As the number of agents increases, centralized control designs fail to guarantee robustness and are harder to implement than decentralized approaches, which also provide a reduce in the computational complexity of the overall feedback scheme.

Among the various objectives that the control design aims to impose on the agents, convergence of the multi-agent team to a common configuration, known as the agreement problem, is a specification that has been extensively pursued recently. Many control laws that achieve distributed agreement have been presented, e.g., [1],[16], [6],[12],[18]. Furthermore, the application of motion models of large populations of animals/insects (swarms) in nature to multiagent systems is also a field of extensive research activity in the last few years. Relevant results include algorithms for swarm aggregation [9] and flocking [15],[20].

This paper presents a control methodology for nonholonomic swarm dispersion which can be considered as an inverse agreement problem. Each agent follows a flow, whose inverse leads the team to agreement [6]. The control is distributed, in the sense that each agent has only knowledge of the states of agents located within its sensing zone at each

Dimos Dimarogonas is with KTH ACCESS Linnaeus Center, School of Electrical Engineering, Royal Institute of Technology (KTH), Stockholm, Sweden \{dimos@ee.kth.se\}. Kostas Kyriakopoulos is with Control Systems Lab, Department of Mechanical Engineering, National Technical University of Athens, Greece \{kkyria@mail.ntua.gr $\}$. The authors were supported by EU through contract I-SWARM (IST-2004507006). The first author'work was also done within TAIS-AURES program (297316-LB704859), funded by the Swedish Governmental Agency for Innovation Systems (VINNOVA) and Swedish Defence Materiel Administration (FMV). He was also supported by the Swedish Research Council, the Swedish Foundation for Strategic Research, and EU NoE HYCON. time instant. The sensing zone is assumed to be a circular area around each agent. The application of this inverse agreement strategy is dispersion of the nonholonomic agents, i.e., convergence to a configuration where the minimum distance between the swarm members is bounded from below by a controllable lower bound. It is shown that this bound coincides with the radius of the sensing zone of the agents. The results are then extended to the case of a circular bounded workspace. Applications of the proposed dispersion algorithm include coverage control [7], [4], and optimal placement of a multi-robot team in small areas [11],[2]. However, we also show that inverse agreement algorithms can be used to provide solutions to various problems in multiagent control. This is a topic of probable related research directions. We note that the results of this paper were shown to hold for the case of single integrator point agents [5].

The rest of the paper is organized as follows: Section II presents the system and problem in hand. Tools from nonsmooth and matrix theory used in the paper are reviewed in Section III. The swarm dispersion methodology is presented in Section IV, while the case of a bounded workspace is considered in Section V. Simulations supporting the theory are included in Section VI. Section VII summarizes the results of this paper and indicates current research efforts.

\section{System AND Problem Description}

Consider $N$ nonholonomic circular agents operating in a workspace $W \subset \mathbb{R}^{2}$. Let $q_{i}=\left[x_{i}, y_{i}\right]^{T} \in \mathbb{R}^{2}$ be the position of agent $i$. All agents have a common radius $r$. However, the proposed framework is easily applied to agents with varying, not necessarily common, radii. The configuration space is spanned by $q=\left[q_{1}^{T}, \ldots, q_{N}^{T}\right]^{T}$. Each agent has an orientation $\theta_{i}$ with respect to the global coordinate frame. The orientation vector of the agents is $\theta=\left[\theta_{1} \ldots \theta_{N}\right]^{T}$. The configuration of each agent is $p_{i}=\left[\begin{array}{ll}q_{i}^{T} & \theta_{i}\end{array}\right]^{T} \in$ $\mathbb{R}^{2} \times(-\pi, \pi]$. Agent motion is described by the kinematics:

$$
\begin{aligned}
& \dot{x}_{i}=u_{i} \cos \theta_{i} \\
& \dot{y}_{i}=u_{i} \sin \theta_{i}, i \in \mathcal{N}=[1, \ldots, N] \\
& \dot{\theta}_{i}=\omega_{i}
\end{aligned}
$$

where $u_{i}, \omega_{i}$ denote the translational and rotational velocity (control inputs) of agent $i$, respectively.

Each agent can only sense agents whose center is found within a circle of radius $d$, common for all agents, around the center of the agent. This circle is called the sensing zone of agent $i$ and $d$ its sensing radius. We require that $d$ is larger than the sum of the radii of any two agents, i.e. $d>2 r$. We denote by $N_{i}$ the subset including the 
agents that $i$ can sense at each time instant, i.e., $N_{i}=$ $\left\{j \in \mathcal{N}, j \neq i:\left\|q_{i}-q_{j}\right\| \leq d\right\}$. The objective is dispersion of the team members in a distributed manner. We assume that a large number of agents is gathered in a workspace close to each other. The goal is to design control laws that force the agents to converge to sufficiently large distances between them. Specifically, we equip each agent with a repulsive potential field with respect to each other agent within its sensing zone. The main result states that the closed loop system converges to a configuration where the sensing zone of each agent is empty, i.e. every agent is located at a distance no less than $d$ from every other agent. In addition, the control law is designed to guarantee collision avoidance between the team members, in the sense that the discs that represent the agent never overlap throughout the closed loop system evolution. The stability analysis is performed first for a bounded and then an unbounded workspace.

A dispersion potential between agents $i$ and $j$ is given by

$$
\gamma_{i j}\left(\beta_{i j}\right)=\left\{\begin{array}{l}
\frac{1}{2} \beta_{i j}, 0 \leq \beta_{i j} \leq c^{2} \\
\phi\left(\beta_{i j}\right), c^{2} \leq \beta_{i j} \leq d^{2} \\
h, d^{2} \leq \beta_{i j}
\end{array}\right.
$$

where $\beta_{i j}=\left\|q_{i}-q_{j}\right\|^{2}-4 r^{2}$. The positive scalars $c, d, h$ and the function $\phi$ are chosen in such a way so that $\gamma_{i j}$ is everywhere $C^{1}$. In this paper, we choose the polynomial function $\phi(x)=a_{2} x^{2}+a_{1} x+a_{0}$. The parameters of $\phi(x)$ satisfy the differentiability requirement for $\gamma_{i j}$, provided that they fulfil the following relations: $a_{2}=\frac{1}{4\left(c^{2}-d^{2}\right)}, a_{1}=\frac{d^{2}}{2\left(d^{2}-c^{2}\right)}$, $a_{0}=\frac{c^{4}}{4\left(c^{2}-d^{2}\right)}, h=\frac{d^{2}+c^{2}}{4}$. The gradient and the partial derivative of $\gamma_{i j}$ are computed by $\nabla \gamma_{i j}=2 \rho_{i j} D_{i j} q$ and $\frac{\partial \gamma_{i j}}{\partial q_{i}}=2 \rho_{i j}\left(D_{i j}\right)_{i} q$ where $\rho_{i j} \triangleq \frac{\partial \gamma_{i j}}{\partial \beta_{i j}}$ and the matrices $D_{i j},\left(D_{i j}\right)_{i}$, for $i<j$ are given by $D_{i j}=\tilde{D}_{i j} \otimes I_{2}$, where $\left(\tilde{D}_{i j}\right)_{i i}=\left(\tilde{D}_{i j}\right)_{j j}=1,\left(\tilde{D}_{i j}\right)_{i j}=\left(\tilde{D}_{i j}\right)_{j i}=-1$ and $\left(\tilde{D}_{i j}\right)_{k l}=0$ for $k, l \neq i, j$, and $\left(D_{i j}\right)_{i}=$ $\left[\begin{array}{lllll}O_{1 \times(i-1)} & 1 & O_{1 \times(j-i-1)} & -1 & O_{1 \times(N-j)}\end{array}\right] \otimes I_{2}$ where $\otimes$ denotes the standard Kronecker product between two matrices [10]. The definition of the matrices $D_{i j},\left(D_{i j}\right)_{i}$, for $i>j$ is straightforward. It can easily be shown that $\rho_{i j}>0$ for $0<\beta_{i j}<d^{2}$ and $\rho_{i j}=0$ for $\beta_{i j} \geq d^{2}$.

\section{MATHEMATICAL PRELIMINARIES}

We review tools from matrix theory and nonsmooth analysis that we use in the analysis of the following paragraphs.

\section{A. Tools from Matrix Theory}

For an undirected graph $\mathcal{G}=(V, E)$ with $n$ vertices denote by $V$ its set of vertices and by $E$ its set of edges. If there is an edge connecting two vertices $i, j$, i.e. $(i, j) \in E$, then $i, j$ are called adjacent. A path of length $r$ from a vertex $i$ to a vertex $j$ is a sequence of $r+1$ distinct vertices starting with $i$ and ending with $j$ such that consecutive vertices are adjacent. If there is a path between any two vertices of the graph $\mathcal{G}$, then $\mathcal{G}$ is called connected. The undirected graph $\mathcal{G}=(V, E)$ corresponding to a real symmetric $n \times n$ matrix
$M$ is a graph with $n$ vertices indexed by $1, \ldots, n$ such that there is an edge between vertices $i, j \in V$ if and only if $M_{i j} \neq 0$, i.e. $(i, j) \in E \Leftrightarrow M_{i j} \neq 0$. A $n \times n$ real symmetric matrix with non-positive off-diagonal elements and zero row sums is called a symmetric Metzler matrix. All the eigenvalues of a symmetric Metzler matrix are nonnegative and zero is a trivial eigenvalue [13]. Zero is a simple eigenvalue of a symmetric Metzler matrix if and only if the corresponding undirected graph is connected. The trivial corresponding eigenvector is the vector of ones, $\overrightarrow{\mathbf{1}}$ [14].

\section{B. Tools from Nonsmooth Analysis}

For a differential equation with discontinuous right-hand side we have the following definition:

Definition 1: [8] In the case of a finite dimensional statespace, the vector function $x($.$) is called a Filippov solution$ of $\dot{x}=f(x)$, where $f$ is measurable and essentially locally bounded, if it is absolutely continuous and $\dot{x} \in K[f](x)$ almost everywhere where $K[f](x) \equiv \overline{c o}\left\{\lim _{x_{i} \rightarrow x} f\left(x_{i}\right) \mid x_{i} \notin\right.$ $N_{0}$ \} and $N_{0}$ is a set of measure zero that contains the set of points where $f$ is not differentiable.

The following chain rule provides a calculus for the time derivative of the energy function in the nonsmooth case:

Theorem 1: [19] Let $x$ be a Filippov solution to $\dot{x}=f(x)$ on an interval containing $t$ and $V: \mathbb{R}^{n} \rightarrow \mathbb{R}$ be a Lipschitz and regular function. Then $V(x(t))$ is absolutely continuous, $(d / d t) V(x(t))$ exists almost everywhere and

$$
\frac{d}{d t} V(x(t)) \in^{a . e \cdot} \dot{\tilde{V}}(x):=\bigcap_{\xi \in \partial V(x(t))} \xi^{T} K[f](x(t))
$$

where "a.e." stands for "almost everywhere".

In this theorem, $\partial V$ is Clarke's generalized gradient. The definition of $\partial V$ and of the regularity of a function are found in [3]. In this paper, the Lyapunov function $V$ is smooth and hence regular, while $\partial V$ is a singleton which is equal to the usual gradient everywhere: $\partial V(x)=\{\nabla V(x)\} \forall x$. We use the following nonsmooth version of LaSalle's principle:

Theorem 2: [19] Let $\Omega$ be a compact set such that every Filippov solution to $\dot{x}=f(x), x(0)=x\left(t_{0}\right)$ starting in $\Omega$ is unique and remains in $\Omega$ for all $t \geq t_{0}$. Let $V: \Omega \rightarrow R$ be a time independent regular function such that $v \leq 0, \forall v \in \dot{\widetilde{V}}$ (if $\dot{\tilde{V}}$ is the empty set then this is trivially satisfied). Define $S=\{x \in \Omega \mid 0 \in \dot{\widetilde{V}}\}$. Then every trajectory in $\Omega$ converges to the largest invariant set, $M$, in the closure of $S$.

\section{SWARM DISPERSION WITH COLLISION AVOIDANCE}

Consider $V=\sum_{i} \sum_{j \neq i} \frac{1}{\gamma_{i j}}$ as a candidate Lyapunov function for the closed loop system. Since $V$ is smooth in the domain of interest, its generalized gradient is a singleton and coincides with its usual gradient. We can compute $\nabla V=$ $\sum_{i} \sum_{j \neq i}\left(-\frac{1}{\gamma_{i j}^{2}}\right) \nabla \gamma_{i j}=-\sum_{i} \sum_{j \neq i} \frac{2 \rho_{i j}}{\gamma_{i j}^{2}} D_{i j} q=-2\left(R_{1} \otimes I_{2}\right) q$, where the matrix $R_{1}$ is given by

$$
\left(R_{1}\right)_{i j}=\left\{\begin{array}{l}
\sum_{j \neq i} \frac{\rho_{i j}}{\gamma_{i j}^{2}}+\sum_{j \neq i} \frac{\rho_{j i}}{\gamma_{j i}^{2}}, i=j \\
-\frac{\rho_{i j}}{\gamma_{i j}^{2}}-\frac{\rho_{j i}^{2}}{\gamma_{j i}^{2}}, i \neq j
\end{array}\right.
$$


Then, $\frac{\rho_{i j}}{\gamma_{i j}^{2}}=\frac{\rho_{j i}}{\gamma_{j i}^{2}} \Rightarrow R_{1}=2 R_{2}$ where

$$
\left(R_{2}\right)_{i j}=\left\{\begin{array}{l}
\sum_{j \neq i} \frac{\rho_{i j}}{\gamma_{i j}^{2}}, i=j \\
-\frac{\rho_{i j}}{\gamma_{i j}^{2}}, i \neq j
\end{array}\right.
$$

and hence $\nabla V=-4\left(R_{2} \otimes I_{2}\right) q$. Denote the stack vector $q=[x, y]^{T}$ into the coefficients that correspond to the $x, y$ directions of the agents respectively, while the notation $(a)_{i}$ denotes the $i$-th element of the vector $a$. We propose the following control law for each agent $i$ :

$$
\begin{gathered}
u_{i}=\operatorname{sgn}\left\{r_{x i} \cos \theta_{i}+r_{y i} \sin \theta_{i}\right\} \cdot\left(r_{x i}^{2}+r_{y i}^{2}\right) \\
\omega_{i}=-\left(\theta_{i}-\arctan 2\left(r_{y i}, r_{x i}\right)\right)
\end{gathered}
$$

where $r_{x i}=\left(R_{2} x\right)_{i}, r_{y i}=\left(R_{2} y\right)_{i}$. We point out that since $\rho_{i j}=0$ for $\beta_{i j} \geq d^{2}$, the proposed control law satisfies the agents' limited sensing radius, since each agent $i$ has to take into account only agents within its sensing zone in order to implement the control laws (2),(3). In particular, we have $r_{x i}=\left(R_{2} x\right)_{i}=\sum_{j \neq i} \frac{2 \rho_{i j}}{\gamma_{i j}^{2}}\left(x_{i}-x_{j}\right)=\sum_{j \in N_{i}} \frac{2 \rho_{i j}}{\gamma_{i j}^{2}}\left(x_{i}-x_{j}\right)$, $r_{y i}=\left(R_{2} y\right)_{i}=\sum_{j \neq i} \frac{2 \rho_{i j}}{\gamma_{i j}^{2}}\left(y_{i}-y_{j}\right)=\sum_{j \in N_{i}} \frac{2 \rho_{i j}}{\gamma_{i j}^{2}}\left(y_{i}-y_{j}\right)$, and hence agent $i$ must be aware of the relative positions only of those agents belonging to $N_{i}$ at each time instant. The generalized time derivative of $V$ is given by:

$$
\begin{gathered}
\dot{\tilde{V}}=(\nabla V)^{T} \cdot K\left[\begin{array}{c}
u_{1} \cos \theta_{1} \\
u_{1} \sin \theta_{1} \\
\vdots \\
u_{N} \cos \theta_{N} \\
u_{N} \sin \theta_{N}
\end{array}\right] \subset \\
{\left[\begin{array}{c}
u_{1} \cos \theta_{1} \\
u_{1} \sin \theta_{1} \\
\vdots \\
\left.C-4\left(\left(R_{2} \otimes I_{2}\right) q\right)^{T} K \begin{array}{c}
u_{N} \cos \theta_{N} \\
u_{N} \sin \theta_{N}
\end{array}\right] \subset \\
\subset-4\left(R_{2} x\right)^{T}\left[\begin{array}{c}
K\left[u_{1}\right] \cos \theta_{1} \\
\vdots \\
K\left[u_{N}\right] \cos \theta_{N}
\end{array}\right]- \\
\left.K\left[u_{1}\right] \sin \theta_{1}\right] \\
\vdots \\
K\left[u_{N}\right] \sin \theta_{N}
\end{array}\right] \subset} \\
\subset-\sum_{i \in \mathcal{N}}\left\{4 K\left[u_{i}\right]\left(r_{x i} \cos \theta_{i}+r_{y i} \sin \theta_{i}\right)\right\}
\end{gathered}
$$

where we used Theorem 1.3, [17] to calculate the inclusions of the Filippov set. Since $K[\operatorname{sgn}(x)] x=$ $\{|x|\}([17]$, Theorem 1.7), the choice of controls (2),(3) yields

$$
\dot{\widetilde{V}}=-\sum_{i}\left\{4\left|r_{x i} \cos \theta_{i}+r_{y i} \sin \theta_{i}\right|\left(r_{x i}^{2}+r_{y i}^{2}\right)\right\} \leq 0
$$

so that the generalized derivative of $V$ reduces to a singleton.

The first result establishes collision avoidance:

Lemma 1: Consider the system of multiple kinematic agents (1) driven by the control law (2),(3) and starting from a feasible set of initial positions $\mathcal{I}(q)=$ $\left\{q \mid\left\|q_{i}-q_{j}\right\|>2 r, \forall i, j \in \mathcal{N}, i \neq j\right\}$. Then the set $\mathcal{I}(q)$ is invariant for the trajectories of the closed loop system.

Proof: For every initial condition $q(0) \in \mathcal{I}(q), \dot{\tilde{V}}$ remains non-positive for all $t \geq 0$, by virtue of (4). Hence $V(q(t)) \leq$ $V(q(0))<\infty$ for all $t \geq 0$. Since $V \rightarrow \infty$ if and only if $\left\|q_{i}-q_{j}\right\| \rightarrow 2 r$ for at least one pair $i, j \in \mathcal{N}$, we conclude that $q(t) \in \mathcal{I}(q)$, for all $t \geq 0$. $\diamond$

By virtue of Lemma 1, collision avoidance is guaranteed. The control design however is also directly related to the final configurations of the nonholonomic swarm members. The main result of this section is summarized in the following:

Theorem 2: Consider the system of multiple nonholonomic agents (1) driven by the control law (2),(3) and starting from a set of initial positions $\mathcal{I}(q) \cap \mathcal{F}(q)$ where $\mathcal{I}(q)=\left\{q \mid\left\|q_{i}-q_{j}\right\|>2 r, \forall i, j \in \mathcal{N}, i \neq j\right\}$ was defined in Lemma 1 and $\mathcal{F}(q)=$ $\left\{q \mid\left\|q_{i}-q_{j}\right\|<(N-1) d^{*}, \forall i, j \in \mathcal{N}, i \neq j,\right\} \quad$ where $d^{*}>d$ is chosen arbitrarily. Then the agents reach a static configuration (i.e. all agents eventually stop) which satisfies $\left\|q_{i}-q_{j}\right\| \geq d, \forall i, j \in \mathcal{N}, i \neq j$, with zero orientations.

Proof: Since the set of initial conditions is contained in $\mathcal{I}(q)$, we have $\left\|q_{i}(t)-q_{j}(t)\right\|>2 r \Rightarrow q_{i}(t) \neq q_{j}(t)$, for all $i, j \in \mathcal{N}, i \neq j$, and all $t \geq 0$, by virtue of Lemma 1. Pick $V$ as a candidate Lyapunov function. The generalized time derivative of $V$ is reduced to a singleton within $\mathcal{I}(q)$, and is given by (4): $\dot{\tilde{V}}=-\sum_{i}\left\{4\left|r_{x i} \cos \theta_{i}+r_{y i} \sin \theta_{i}\right|\left(r_{x i}^{2}+r_{y i}^{2}\right)\right\} \leq 0$. The boundedness of solutions of the closed-loop system is checked based on the relative positions of the swarm members. Pick $d^{*}>d$. Since $\rho_{i j}=0$ whenever $\beta_{i j}>d$, the set $\left\|q_{i}-q_{j}\right\| \leq(N-1) d^{*}$ for all $i, j \in \mathcal{N}$ is positively invariant for the trajectories of the closed-loop system. By virtue of Lemma $1, \mathcal{I}(q) \cap \mathcal{F}(q)$ is also positively invariant. Since this set is closed and bounded, we can apply Theorem 2, to deduce that the agents converge to the largest invariant subset of the set $S=$ $\left\{\left(r_{x i}=r_{y i}=0\right) \vee\left(r_{x i} \cos \theta_{i}+r_{y i} \sin \theta_{i}=0\right), \forall i \in N\right\}$.

However, for each $i \in \mathcal{N}$, we have $\left|\omega_{i}\right|=\frac{\pi}{2}$ whenever $r_{x i} \cos \theta_{i}+r_{y i} \sin \theta_{i}=0$, due to (3). In particular, (3) renders the surface $r_{x i} \cos \theta_{i}+r_{y i} \sin \theta_{i}=0$ noninvariant for agent $i$, whenever $i$ is not located at the desired equilibrium, namely when $r_{x i}=r_{y i}=0$. Hence the largest invariant set $S_{v}$ contained in $S$ is $S \supset S_{v}=\left\{r_{x i}=r_{y i}=0, \forall i \in \mathcal{N}\right\}$. Within $S_{v}$, we have $r_{x i}=r_{y i}=0, \forall i \in \mathcal{N}$ and hence $u_{i}=0$, for all $i \in \mathcal{N}$, i.e., all agents eventually stop. Moreover, within $S_{v}$, we have $\dot{\theta}_{i}=-\theta_{i}$ for all $i$, and hence the orientations of all agents tend to zero. Note now $r_{x i}=r_{y i}=0, \forall i \in \mathcal{N}$ is equivalent to $r_{x i}=r_{y i}=0, \forall i \in \mathcal{N} \Rightarrow\left(R_{2} \otimes I_{2}\right) q=0$. Hence $S_{v}$ can be written as $S_{v}=\left\{q \mid\left(R_{2} \otimes I_{2}\right) q=0\right\}$. We next show that $S_{v}$ can only be invariant when it coincides with the set $S_{0}=\left\{q \mid \rho_{i j}=0, \forall i, j \in \mathcal{N}, i \neq j\right\}$. Clearly, $S_{0}$ is a subset of $S_{v}$ which is invariant for the trajectories of the closed-loop system. Suppose now that $\rho_{i j}>0$ for some pairs of the team members. We denote the undirected graph corresponding to $R_{2}$ by $\mathcal{G}\left(R_{2}\right)$. The assumption that $\rho_{i j}>0$ for some pairs 
$i, j \in \mathcal{N}, i \neq j$ guarantees that $\mathcal{G}\left(R_{2}\right)$ has at least one edge. The graph $\mathcal{G}\left(R_{2}\right)$ can now be decomposed into its connected components. Please note that since the graph is undirected, no vertex can belong to two different components simultaneously. Ignoring the connected components containing only one vertex (i.e. vertices $k$ for which $\rho_{k j}=0$ for all $j \neq k$ ), and rearranging the agent indices accordingly, equation $\left(R_{2} \otimes I_{2}\right) q=0$ can be decomposed into different equations, each of which corresponds to a different connected component of $\mathcal{G}\left(R_{2}\right)$. Specifically for the connected component containing agents/vertices $\left\{i_{1}, i_{2}, \ldots, i_{l}\right\}, i_{j} \in \mathcal{N}, j=1, \ldots l$ with $l \leq n$ we have $\left(\tilde{R}_{2} \otimes I_{2}\right) \tilde{q}=0$, where $\tilde{q}=\left[\begin{array}{lll}q_{i_{1}}^{T} & \ldots & q_{i_{l}}^{T}\end{array}\right]^{T}$ and the $l \times l$ matrix $\tilde{R}_{2}$ has the same form as $R_{2}$ taking into account the set of agents $\left\{i_{1}, i_{2}, \ldots, i_{l}\right\}$. By denoting $\tilde{x}, \tilde{y}$ the stack vectors of $\tilde{q}$ in the $x, y$ directions, we have $\left(\tilde{R}_{2} \otimes I_{2}\right) \tilde{q}=0 \Rightarrow \tilde{R}_{2} \tilde{x}=\tilde{R}_{2} \tilde{y}=0$. Matrix $\tilde{R}_{2}$ has zero row sums and non-positive off-diagonal elements, i.e., it is a symmetric Metzler matrix. Thus, the eigenvalues of $\tilde{R}_{2}$ are nonnegative. Furthermore, since $\tilde{R}_{2}$ corresponds to a connected graph (a connected component of $\mathcal{G}\left(R_{2}\right)$ ), zero is a simple eigenvalue of $\tilde{R}_{2}$ with corresponding eigenvector the vector of ones, $\overrightarrow{\mathbf{1}}$. Hence equations $\tilde{R}_{2} \tilde{x}=\tilde{R}_{2} \tilde{y}=0$ guarantee that both $\tilde{x}, \tilde{y}$ are eigenvectors of $\tilde{R}_{2}$ belonging to $\operatorname{span}\{\overrightarrow{\mathbf{1}}\}$. Thus, all elements of $\tilde{q}$ attain the same value, implying that all agents converge to a common configuration at steady state. However this is impossible, since, due to the invariance of $\mathcal{I}(q)$, no trajectory of the closed-loop system starting from $\mathcal{I}(q)$ can ever leave this set, i.e., $\left\|q_{i}(t)-q_{j}(t)\right\|>2 r$, and hence $q_{i}(t) \neq q_{j}(t)$, for all $i, j \in \mathcal{N}, i \neq j$, and all $t \geq 0$. We conclude that the largest invariant subset of $S$ is $S_{0}$. Since $\rho_{i j}=0$ only for $\left\|q_{i}-q_{j}\right\| \geq d$, the proof is complete. $\diamond$

Thus, the closed-loop system converges to a configuration where each agent is located at a distance no less than $d$ from every other agent. This reveals an important geometric property: since any pair of agents is located at least at a distance $d$ from each other, each agent occupies a disc of radius $d / 2$ in which no other agent is present. In other words, the agents are dispersed to $n$ disjoint discs of radius $d / 2$.

\section{The Bounded Workspace Case}

We proposed a dispersion algorithm for multiple nonholonomic agents in an unbounded workspace. In practical applications such as coverage control and sensor deployment the problem is to modify the controller in order to take into account the workspace boundary. In this paper, we consider the case of a cyclic workspace $W_{0}$ of radius $R_{W_{0}}$. However, the proposed design is applicable to any convex workspace. In order to take into account the agent radii, we define a virtual workspace $W$ in the interior of $W_{0}$ that has the same center as $W_{0}$ and radius $R_{W}=R_{W_{0}}-r<R_{W_{0}}$ (see Figure 1 ). By proving that the center of each agent $q_{i}$ remains within $W$ for all time it is also guaranteed that the discs representing the agents remain within the real workspace $W_{0}$. Hence we consider the virtual workspace $W$ in the sequel.

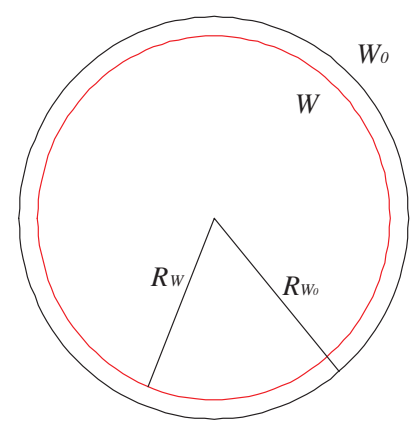

Fig. 1. The virtual boundary $W$ is a smaller circle in the interior of the real boundary $W_{0}$ with the same center and radius equal to $R_{W}=R_{W_{0}}-r$.

A potential similar to the inter-agent dispersion potential is used for the agent-boundary repulsion. Copying with the sensing capabilities of the agents, the repulsive potential of each agent with respect to the workspace boundary is defined as

$$
\gamma_{i b}\left(\beta_{i b}\right)=\left\{\begin{array}{l}
\frac{1}{2} \beta_{i b}, 0 \leq \beta_{i b} \leq c_{b}^{2} \\
\varphi_{b}\left(\beta_{i b}\right), c_{b}^{2} \leq \beta_{i b} \leq d_{b}^{2} \\
h_{b}, d_{b}^{2} \leq \beta_{i b}
\end{array}\right.
$$

where $\beta_{i b}=\left\|q_{i}-q_{i, \min }\right\|^{2}, d_{b}<d$ and $q_{i, \min }=$ $\arg \min _{q \in \partial W}\left\|q_{i}-q\right\|^{2}$. Note that $q_{i, \min }$ is continuous for all $i$ due to the convexity of $W$. The positive scalars $h_{b}, c_{b}$ and the function $\varphi_{b}$ are defined in such a way so that $\gamma_{i b}$ is rendered everywhere continuously differentiable. Each agent has to have knowledge of the workspace boundary only when located at a distance smaller than $d_{b}$ from it.

Denote $\rho_{i b}=\frac{\partial \gamma_{i b}}{\partial \beta_{i b}}$. We have $\rho_{i b}=0$ for $\beta_{i b}>d_{b}^{2}$ and $\rho_{i b}>0$ for $\beta_{i b} \leq d_{b}^{2}$. Denote by $q_{\min }$ the stack vector of all $q_{\min }^{i}$ and by $x_{\min }, y_{\min }$ its coefficients in the $x, y$ directions. Similarly to the case of an unbounded workspace, we use $V_{b}=\sum_{i} \sum_{j \neq i} \frac{1}{\gamma_{i j}}+\sum_{i} \frac{1}{\gamma_{i b}}$ as a candidate Lyapunov function. Since $V_{b}$ is smooth in the domain of interest, $\partial V_{b}$ is a singleton and coincides with its usual gradient. The latter is calculated by $\nabla V_{b}=-4\left(R_{3} \otimes I_{2}\right) q+$ $4\left(R_{4} \otimes I_{2}\right) q_{\min }$ where $R_{3}=R_{2}+\operatorname{diag}\left\{\frac{\rho_{1 b}}{\gamma_{1 b}^{2}}, \ldots, \frac{\rho_{N b}}{\gamma_{N b}^{2}}\right\}$ and $R_{4}=\operatorname{diag}\left\{\frac{\rho_{1 b}}{\gamma_{1 b}^{2}}, \ldots, \frac{\rho_{N b}}{\gamma_{N b}^{2}}\right\}$. We propose the following control law for each agent $i$ of the nonholonomic swarm

$$
\begin{gathered}
u_{i}=\operatorname{sgn}\left\{r_{x i}^{b} \cos \theta_{i}+r_{y i}^{b} \sin \theta_{i}\right\} \cdot\left(\left(r_{x i}^{b}\right)^{2}+\left(r_{y i}^{b}\right)^{2}\right) \\
\omega_{i}=-\left(\theta_{i}-\arctan 2\left(r_{y i}^{b}, r_{x i}^{b}\right)\right)
\end{gathered}
$$

where $r_{x i}^{b}=\left(R_{3} x\right)_{i}-\left(R_{4} x_{\min }\right)_{i}, r_{y i}^{b}=\left(R_{3} y\right)_{i}-\left(R_{4} y_{\min }\right)_{i}$.

The generalized time derivative of $V_{b}$ is now given by

$$
\dot{\tilde{V}_{b}}=\left(\nabla V_{b}\right)^{T} \cdot K\left[\begin{array}{c}
u_{1} \cos \theta_{1} \\
u_{1} \sin \theta_{1} \\
\vdots \\
u_{N} \cos \theta_{N} \\
u_{N} \sin \theta_{N}
\end{array}\right] \subset
$$




$$
-4\left(\left(R_{3} \otimes I_{2}\right) q-\left(R_{4} \otimes I_{2}\right) q_{\min }\right)^{T}\left[\begin{array}{c}
K\left[u_{1}\right] \cos \theta_{1} \\
K\left[u_{1}\right] \sin \theta_{1} \\
\vdots \\
K\left[u_{N}\right] \cos \theta_{N} \\
K\left[u_{N}\right] \sin \theta_{N}
\end{array}\right]
$$$$
\subset-4\left(R_{3} x-R_{4} x_{\min }\right)^{T}\left[\begin{array}{c}
K\left[u_{1}\right] \cos \theta_{1} \\
\vdots \\
K\left[u_{N}\right] \cos \theta_{N}
\end{array}\right]
$$$$
-4\left(R_{3} y-R_{4} y_{\min }\right)^{T}\left[\begin{array}{c}
K\left[u_{1}\right] \sin \theta_{1} \\
\vdots \\
K\left[u_{N}\right] \sin \theta_{N}
\end{array}\right]
$$$$
\subset-\sum_{i}\left\{4 K\left[u_{i}\right]\left(r_{x i}^{b} \cos \theta_{i}+r_{y i}^{b} \sin \theta_{i}\right)\right\}
$$

The choice of control laws (5),(6) now yields

$$
\dot{\tilde{V}}_{b}=-\sum_{i}\left\{4\left|r_{x i}^{b} \cos \theta_{i}+r_{y i}^{b} \sin \theta_{i}\right|\left(\left(r_{x i}^{b}\right)^{2}+\left(r_{y i}^{b}\right)^{2}\right)\right\}
$$

so that $\dot{\tilde{V}}_{b}$ reduces again to a singleton. The first result guarantees that the interior of the workspace is invariant:

Lemma 3: Consider (1) driven by (5),(6) and starting from the set of initial conditions $\mathcal{I}(q) \cap \mathcal{J}(q)$ where $\mathcal{J}(q)=$ $\left\{q \mid q_{i} \in \operatorname{int}(W) \triangleq W \backslash \partial W, \forall i \in \mathcal{N}\right\}$ is the interior of $W$ and $\mathcal{I}(q)$ was defined previously. Then $\mathcal{I}(q) \cap \mathcal{J}(q)$ is invariant for the trajectories of the closed-loop system.

Proof: $\mathcal{I}(q)$ is invariant by Lemma 1. For every initial condition $q(0) \in \mathcal{I}(q) \cap \mathcal{J}(q), \dot{\tilde{V}}_{b}$ remains non-positive for all $t \geq 0$, by virtue of (7). Hence $V_{b}(q(t)) \leq V_{b}(q(0))<\infty$ for all $t \geq 0$. Since $V_{b} \rightarrow \infty$ whenever $q_{i} \rightarrow q_{i, \min }$ for at least one agent $i \in \mathcal{N}$, and the latter implies $q_{i} \rightarrow \partial W$, we conclude that $q(t) \in \mathcal{J}(q)$, for all $t \geq 0$. $\diamond$

Thus, if agents start within the interior of the virtual workspace, they are forced to remain within it. Collision avoidance, i.e., Lemma 1 also holds. We now show that agents reach a configuration where $u_{i}=0, \theta_{i}=0$ for all $i$ :

Corollary 4: Consider the system of multiple nonholonomic agents (1) driven by (5),(6) and starting from the set of initial conditions $\mathcal{I}(q) \cap \mathcal{J}(q)$. Then the system reaches a configuration in which $u_{i}=0$ and $\theta_{i}=0$ for all $i \in \mathcal{N}$.

Proof: $\mathcal{J}(q)$ is closed and bounded for the trajectories of the closed-loop. system, by Lemma 3, and (7) guarantees that $\dot{\tilde{V}}_{b}$ is negative semidefinite. By Theorem 2, the trajectories of the closed-loop system reach the largest invariant subset of the set $S^{b}=$ $\left\{\left(r_{x i}^{b}=r_{y i}^{b}=0\right) \vee\left(r_{x i}^{b} \cos \theta_{i}+r_{y i}^{b} \sin \theta_{i}=0\right), \forall i \in \mathcal{N}\right\}$.

Using the same arguments as in the proof of Theorem 2, we deduce that the largest invariant set $S_{v}^{b}$ contained in $S^{b}$ is $S^{b} \supset S_{v}^{b}=\left\{r_{x i}^{b}=r_{y i}^{b}=0, \forall i \in \mathcal{N}\right\}$. Within $S_{v}^{b}$, we have $r_{x i}^{b}=r_{y i}^{b}=0, \forall i \in \mathcal{N}$ and hence $u_{i}=0$, for all $i \in \mathcal{N}$. Within $S_{v}^{b}$, we also have $\dot{\theta}_{i}=-\theta_{i}$ for all $i$, and hence the orientations of all agents tend to zero. $\diamond$

Note that $r_{x i}^{b}=r_{y i}^{b}=0, \forall i \in \mathcal{N} \Rightarrow\left(R_{3} \otimes I_{2}\right) q-$ $\left(R_{4} \otimes I_{2}\right) q_{\text {min }}=0$. We now show that the control law is related to the final relative positions of the agents in a manner similar to the unbounded case. From the proof of Corollary
4 we derive that the system converges to the largest invariant subset of the set $S_{v}^{b}$. Please note that the result of Lemma 3 holds for arbitrarily small $c_{b}, d_{b}$. For $c_{b}, d_{b} \rightarrow 0$, we have that either $q_{i} \rightarrow q_{i, \min }$, or $\rho_{i b} \rightarrow 0$, for those agents that do not satisfy the condition $q_{i} \rightarrow q_{i, \min }$. Thus, in this case

$$
\begin{aligned}
& \left(R_{3} \otimes I_{2}\right) q-\left(R_{4} \otimes I_{2}\right) q_{\min }= \\
& =\left(R_{2} \otimes I_{2}\right) q-\left(R_{4} \otimes I_{2}\right)\left(q-q_{\min }\right) \\
& =\left(R_{2} \otimes I_{2}\right) q-\left(\left(\operatorname{diag}\left\{\frac{\rho_{1 b}}{\gamma_{1 b}^{2}}, \ldots, \frac{\rho_{N b}}{\gamma_{N b}^{2}}\right\}\right) \otimes I_{2}\right)\left(q-q_{\min }\right) \\
& =\left(R_{2} \otimes I_{2}\right) q- \\
& -\left[\begin{array}{lll}
\frac{\rho_{1 b}}{\gamma_{1 b}^{2}}\left(q_{1}-q_{1, \min }\right) & \ldots & \frac{\rho_{1 b}}{\gamma_{1 b}^{2}}\left(q_{N}-q_{N, \min }\right)
\end{array}\right]^{T} \\
& =\left(R_{2} \otimes I_{2}\right) q
\end{aligned}
$$

since for each $i \in \mathcal{N}$, we have either $q_{i} \rightarrow q_{i, \min }$, or $\rho_{i b} \rightarrow 0$, for $c_{b}, d_{b} \rightarrow 0$ as discussed above. Thus, $S_{v}^{b}$ coincides with the set $S_{v}$ of the proof of Theorem 2 . As proved in that Theorem, the largest invariant subset within $S_{v}$ is the set $S_{0}=\left\{q \mid \rho_{i j}=0, \forall i, j \in \mathcal{N}, i \neq j\right\}=$ $\left\{q \mid\left\|q_{i}-q_{j}\right\| \geq d, \forall i, j \in \mathcal{N}, i \neq j\right\}$. Hence the system reaches a configuration it which all agents remain within the workspace bounds and each agent is located at a distance no less than $d$ from every other agent in the group, provided that such configuration exists within the workspace bounds.

This result is formally stated in the next Theorem:

Theorem 5: Consider the system (1) driven by the controls (5),(6) and starting from $\mathcal{I}(q) \cap \mathcal{J}(q)$. Assume that the set $B(q)=\left\{q \in \operatorname{int}(W) \mid\left\|q_{i}-q_{j}\right\| \geq d, \forall i, j \in \mathcal{N}, i \neq j\right\}$ is nonempty. Then the system reaches a configuration in which all agents remain in the interior of the workspace, and $\left\|q_{i}-q_{j}\right\| \geq d, \forall i, j \in \mathcal{N}, i \neq j$, with zero orientations.

Remark 1: Similarly to the unbounded case, $B(q)$ being non-empty corresponds to a case where each agent occupies a $d / 2$-disc at steady state. If $B(q)$ is empty, the workspace is not large enough to fulfill the above geometric condition, and the system converges to a configuration that minimizes the cost function $V_{b}$, respecting the constraint of Lemma 3 that the agents are forced to remain within the workspace boundary. Thus, some of the $d / 2$-discs may overlap.

Remark 2: Since the centers of agents remain within the virtual workspace boundary, the actual discs that represent the agents remain within the real workspace boundary $\partial W_{0}$.

Remark 3: The results can be extended to the case where the workspace is an arbitrary convex region.

\section{Simulations}

To support the results presented in the previous paragraphs, we provide a series of computer simulations.

In the first simulation, six nonholonomic agents navigate under the controls (2),(3). Screenshots I-III in Figure 2 show the evolution of the closed-loop system. The agents are located at their initial positions in the first screenshot. The sensing radius $d$ is equal to 0.0894 in this simulation. The collision avoidance objective is fulfilled, due to the proposed control design. The agents disperse in the workspace and eventually stop in screenshot III. Screenshot IV depicts the final positions of agents. Each agent occupies a disc of radius $d / 2$. These discs are visualized in Screenshot IV by the 
large discs whose center is the center of corresponding agent. By virtue of Theorem 2, the large discs are disjoint. In the
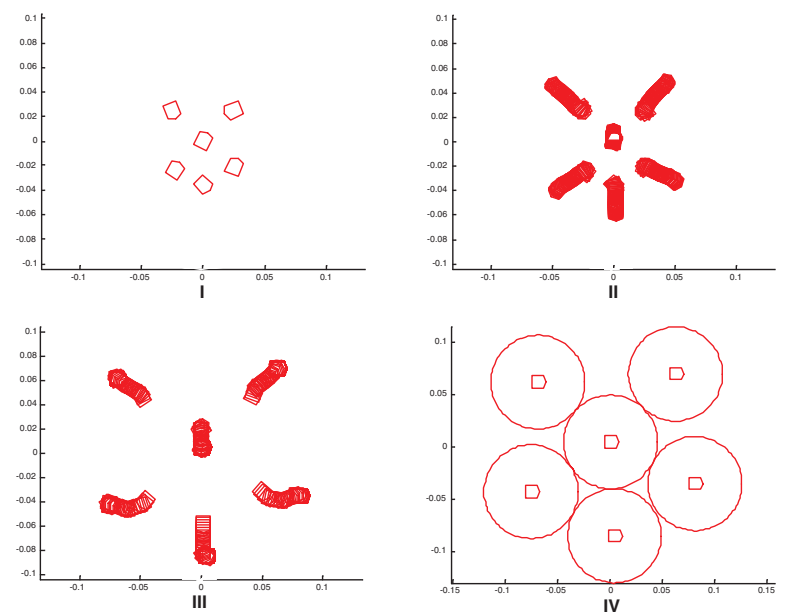

Fig. 2. Swarm dispersion for six nonholonomic agents. The agents disperse in the workspace and eventually occupy six disjoint discs of radius $d / 2$.

second simulation (Figure 3), agents navigate in a bounded workspace under (5),(6). We have again $d=0.0894$ and the workspace radius is $R_{W}=15 d$. Agents start from an initial condition where they are aggregated near the workspace center. Some agents approach the workspace boundary and remain within it due to the repulsive potential. Collision avoidance is fulfilled throughout the closed-loop system evolution. The workspace is large enough to allow the agents to occupy six disjoint discs of radius $d / 2$ at steady state, i.e. the set $B$ of Theorem 5 is nonempty. This is depicted in the last screenshot of Figure 3.
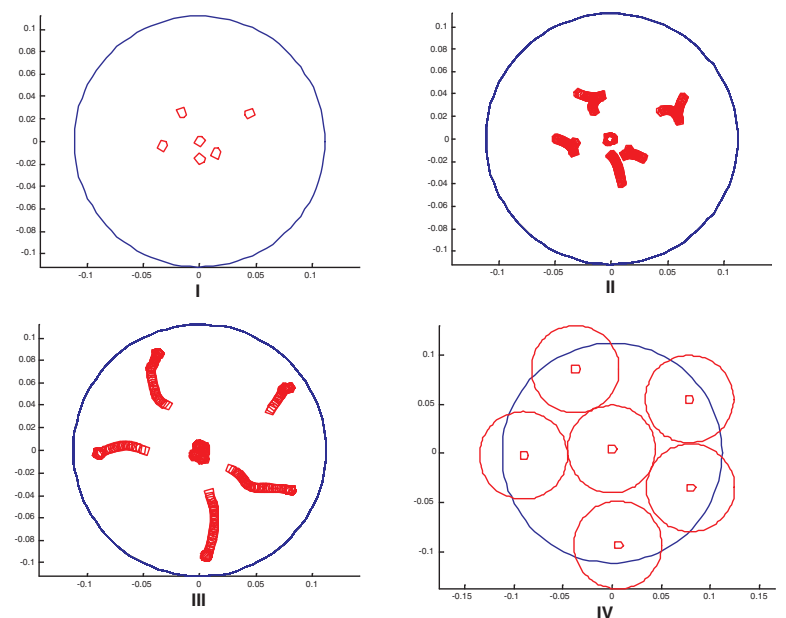

Fig. 3. Swarm dispersion for six nonholonomic agents in a bounded workspace. The workspace is large enough to allow the agents to occupy six disjoint discs of radius $d / 2$ at steady state. Agents are forced to remain within the workspace boundary.

\section{CONCLUSIONS}

We proposed a distributed inverse agreement strategy for multiple nonholonomic agents that forces the agents to disperse in the workspace. Both the cases of unbounded and circular workspace were treated. In the first case, we showed that the closed-loop system reaches a configuration in which the minimum distance between any pair of agents is larger than a specific lower bound. It was proven that this bound coincides with the agents' sensing radius. In the case of a bounded workspace, the control law was redefined in order to force the agents to remain within the workspace boundary throughout the closed loop system evolution. The results were supported through computer simulations. Current research involves exploring the relation of the sensing radius, the number of agents and the radius of the workspace with the emptiness of the set $B$ of Theorem 5 .

\section{REFERENCES}

[1] M. Arcak. Passivity as a design tool for group coordination. IEEE Transactions on Automatic Control, 52(8):1380-1390, 2007.

[2] A. Arsie and E. Frazzoli. Efficient routing of multiple vehicles with no communications. International Journal of Robust and Nonlinear Control, 18(2):154-164, 2007.

[3] F. Clarke. Optimization and Nonsmooth Analysis. Addison - Wesley, 1983.

[4] J. Cortes, S. Martinez, T. Karatas, and F. Bullo. Coverage control for mobile sensing networks. IEEE Transactions on Robotics and Automation, 20(2):243-255, 2004.

[5] D.V. Dimarogonas and K.J. Kyriakopoulos. An inverse agreement control strategy with application to swarm dispersion. 46th IEEE Conf. Decision and Control, pages 6148-6153, 2007.

[6] D.V. Dimarogonas and K.J. Kyriakopoulos. On the rendezvous problem for multiple nonholonomic agents. IEEE Transactions on Automatic Control, 52(5):916-922, 2007.

[7] J. Feddema and D. Schoenwald. Decentralized control of cooperative robotic vehicles. IEEE Transactions on Robotics, 18(5):852-864, 2002.

[8] A. Filippov. Differential equations with discontinuous right-hand sides. Kluwer Academic Publishers, 1988.

[9] V. Gazi and K.M. Passino. Stability analysis of swarms. IEEE Transactions on Automatic Control, 48(4):692-696, 2003.

[10] R. A. Horn and C. R. Johnson. Matrix Analysis. Cambridge University Press, 1996.

[11] Project ISWARM. http://microrobotics.ira.uka.de/.

[12] A. Jadbabaie, J. Lin, and A.S. Morse. Coordination of groups of mobile autonomous agents using nearest neighbor rules. IEEE Transactions on Automatic Control, 48(6):988-1001, 2003.

[13] D.G. Luenberger. Introduction to Dynamic Systems: Theory, Models and Applications. John Wiley \& Sons, 1979.

[14] L. Moreau. Stability of continuous-time distributed consensus algorithms. 43rd IEEE Conf. Decision and Control, pages 3998-4003, 2004.

[15] R. Olfati-Saber. Flocking for multi-agent dynamic systems: Algorithms and theory. IEEE Transactions on Automatic Control, 51(3):401-420, 2006.

[16] R. Olfati-Saber and R.M. Murray. Consensus problems in networks of agents with switching topology and time-delays. IEEE Transactions on Automatic Control, 49(9):1520-1533, 2004.

[17] B. Paden and S. S. Sastry. A calculus for computing Filippov's differential inclusion with application to the variable structure control of robot manipulators. IEEE Trans. on Circuits and Systems, 34(1):73$82,1987$.

[18] W. Ren and R. Beard. Consensus seeking in multiagent systems under dynamically changing interaction topologies. IEEE Transactions on Automatic Control, 50(5):655-661, 2005.

[19] D. Shevitz and B. Paden. Lyapunov stability theory of nonsmooth systems. IEEE Trans. on Automatic Control, 49(9):1910-1914, 1994.

[20] H.G. Tanner, A. Jadbabaie, and G.J. Pappas. Flocking in fixed and switching networks. IEEE Transactions on Automatic Control, 52(5):863-868, 2007. 